\title{
Controle biológico de nematóides gastrintestinais de caprinos em clima semi-árido pelo fungo Monacrosporium thaumasium
}

\author{
Jackson Victor de Araújo(1), Maria de Lurdes de Azevedo Rodrigues ${ }^{(2)}$, Wilson Wouflan Silva(3) \\ e Luiz da Silva Vieira(4)
}

\begin{abstract}
(1)Universidade Federal de Viçosa, Dep. de Veterinária, CEP 36570-000 Viçosa, MG. E-mail: jvictor@ufv.br (2)Universidade Federal Rural do Rio de Janeiro, Instituto de Veterinária, BR 465, Km 7, CEP 23890-000 Seropédica, RJ. E-mail: lurdesar@ufrrj.br (3)Universidade Federal de Campina Grande, Dep. de Medicina Veterinária, CEP 58108-110 Patos, PB. E-mail: wouflan@hotmail.com (4)Embrapa Caprinos, SobralGroaíras, Km 04, Fazenda Três Lagoas, CEP 62011-970 Sobral, CE. E-mail: Ivieira@cnpc.embrapa.br
\end{abstract}

\begin{abstract}
Resumo - O objetivo deste trabalho foi avaliar a viabilidade do fungo nematófago Monacrosporium thaumasium, no controle de larvas infectantes de nematóides gastrintestinais de caprinos em campo, no Semi-Árido cearense. Uma área de 20 ha, com pasto recém-formado, livre de vermes, foi dividida em quatro piquetes de 5 ha. Em cada piquete, foi formado um grupo constituído por nove caprinos, onde cada animal recebeu os seguintes tratamentos, durante os meses de abril a junho de 2002: grupo 1,10 g de péletes por via oral, semanalmente; grupo 2,10 g de péletes por via oral, quinzenalmente; grupo 3, $0,5 \mathrm{~mL}$ de moxidectin por $25 \mathrm{~kg}$ de peso vivo; grupo 4, controle, não tratado. Mensalmente, dois caprinos traçadores foram colocados junto ao rebanho por 30 dias, e após esse período, foram sacrificados e necropsiados. O grupo de animais que recebeu o tratamento com o fungo uma vez por semana apresentou redução no número de ovos por grama de fezes, menor carga parasitária e maior ganho de peso, em relação aos animais dos demais grupos. O fungo M. thaumasium é eficiente no controle de larvas infectantes nas pastagens e pode ser utilizado no controle e profilaxia de nematóides gastrintestinais de caprinos, no Semi-Árido cearense.
\end{abstract}

Termos para indexação: antagonistas fúngicos, controle biológico, fungos nematófagos.

\section{Biological control of gastrointestinal nematodes of goats in semiarid climate by Monacrosporium thaumasium}

\begin{abstract}
The present study aimed to evaluate the viability of the nematophagous fungus Monacrosporium thaumasium in the control of gastrointestinal nematodes larvae of goats in the field, in semiarid climate in Ceará State, Brazil. An area of 20 ha, free of worms, was divided into four paddocks of 5 ha. In each paddock, one group consisting of nine goats was formed and received the following treatment, individually, from April to June 2002: group 1, each animal received, orally, $10 \mathrm{~g}$ of pellets, weekly; group 2, the animals received $10 \mathrm{~g}$ of pellets, each 15 days; group 3, the animals received $0.5 \mathrm{~mL}$ moxidectin per $25 \mathrm{~kg}$ of live weight; group 4, control, not treated. Monthly, two tracer goats were introduced into the flock per 30 days. After this period, they were sacrificed and submitted to necropsy. The group of animals that received fungi treatments weekly showed reduction in the number of eggs per gram of feces, smaller parasitic load and larger weight in relation to animals of the other groups. The fungi $M$. thaumasium was efficient to control infective larvae in the pasture, and can be used in the control and prophylaxis of gastrointestinal nematodes of goats in semiarid climate, in Ceará State.
\end{abstract}

Index terms: fungal antagonists, biological control, nematophagous fungi.

\section{Introdução}

Entre os fatores que interferem na produção de pequenos ruminantes, as helmintoses ocupam grande destaque por causar retardamento do desenvolvimento dos animais (Over et al., 1992), o que resulta em baixa produtividade e, conseqüentemente, elevadas perdas econômicas. Nos trópicos, $95 \%$ dos caprinos estão infectados, principalmente por Haemonchus contortus e Trichostrongylus spp., com taxa de mortalidade acima de 40\% (Githigia et al., 2001).

O controle parasitário na Região do Semi-Árido é baseado no uso de anti-helmínticos, cuja aplicação freqüente a animais de pastejo tem causado resistência 
parasitária em muitos países (Waller et al., 1996; Taylor et al., 2002), inclusive no Brasil (Echevarria et al., 1996).

No Ceará, Melo et al. (2003) constataram, em fazendas comerciais, que várias espécies de nematóides gastrintestinais de caprinos são resistentes ao oxfendazol, levamisol e ivermectina; em algumas situações, seus resíduos decorrentes da metabolização, presentes na carne, leite e excretas dos animais, podem ocasionar problemas de saúde pública e ao ambiente (Mota et al., 2003).

Várias alternativas para controle de nematóides gastrintestinais têm sido desenvolvidas, em resposta ao aumento da resistência aos anti-helmínticos (Hay et al., 1997). O uso de fungos para o controle das verminoses é uma alternativa promissora (Alves et al., 2003; Araújo et al., 2004, 2006). Fungos antagonistas de nematóides propagam-se nas fezes e produzem estruturas especializadas (armadilhas) com a finalidade de capturar e fixar os nematóides (Mota et al., 2003). Após a fixação, o fungo penetra no interior da presa, matandoa por destruição dos seus órgãos internos.

Diversos gêneros de fungos nematófagos, entre eles o gênero Monacrosporium, têm sido utilizados no controle biológico das helmintoses (Gomes et al., 1999; Araújo et al., 2000b). A capacidade do Monacrosporium thaumasium (Drechsler, 1937) em sobreviver à passagem pelo trato gastrintestinal de ruminantes, sem perder a atividade predatória, é um prérequisito importante no controle biológico das helmintoses (Araújo et al., 1999, 2000b; Melo et al., 2003).

O objetivo deste trabalho foi avaliar a viabilidade do fungo nematófago $M$. thaumasium, no controle de larvas infectantes de nematóides gastrintestinais de caprinos em campo, no Semi-Árido nordestino.

\section{Material e Métodos}

O experimento foi realizado de abril a junho de 2002, na Fazenda Três Lagos, da Embrapa Caprinos, no Município de Sobral, Ceará, a $3^{\circ} 42^{\prime} \mathrm{S}$ e $40^{\circ} 2^{\prime} \mathrm{W}$, com altitude de $83 \mathrm{~m}$. A região possui clima semiárido, com duas estações: chuvosa, quando ocorre em média $98,6 \%$ da precipitação anual, e seca. Não houve variação significativa na temperatura, durante todo o ano; a média anual foi de $26,8^{\circ} \mathrm{C}$ (mínima de $25,5^{\circ} \mathrm{C}$ e máxima de $28,9^{\circ} \mathrm{C}$ ). $\mathrm{O}$ índice pluviométrico, registrado durante o período do experimento, foi de $128,1,93,7$ e $40,9 \mathrm{~mm}^{3}$, em abril, maio e junho, respectivamente. Os dados meteororológicos foram coletados mensalmente, em estação especializada da Embrapa Caprinos.

A pastagem nativa foi predominantemente formada pelas espécies: marmeleiro (Croton sonderianus Muell. Arg.), pereiro (Aspidosperma pyrifolium Mart.), jurema-branca (Pithecolobium dumosum Benth.), juazeiro (Zizyphus joazeiro Mart.), pau-branco (Auxemma oncocalyx Taub.), mororó (Bauhinia cheilantha (Bong.) D. Dietr.) e mofumbo (Combretum leprosum Mart.).

Foram utilizados 36 animais permanentes, dos quais 16 fêmeas adultas SRD, adultas e 20 caprinos machos jovens, com idade média de oito meses, da raça AngloNubiano. Trinta dias antes do início do experimento, os animais receberam o vermífugo oxfendazol $(7,5 \mathrm{mg}$ por kg de peso vivo). Sete dias após a vermifugação, foi feita a contagem de ovos por grama de fezes (OPG) (Gordon \& Whitlock, 1939), com três exames realizados na mesma amostra de fezes, e todos os animais tiveram resultado negativo; foram, então, infectados por via oral com aproximadamente 2.000 larvas por dia, de nematóides gastrintestinais (Haemonchus contortus $66 \%$, Trichostrongylus spp. 21\%, Oesophagostomum columbianum 6\% e Strongyloides papillosus 7\%), durante três dias alternados. Os animais permaneceram em estábulo durante 21 dias após a última inoculação de larvas.

Em seguida, uma área de 20 ha, livre de nematóides, foi dividida em quatro piquetes de 5 ha. Foram formados grupos de animais permanentes, constituídos por quatro fêmeas SRD e cinco machos anglo-nubianos. A contagem de OPG inicial dos animais variou de 0 a 200, com média de 100 OPG por grupo de animais.

Péletes do fungo M. thaumasium, obtido de solo brasileiro, foram confeccionados (Araújo et al., 2000a) e continham, em média, 2-2,5 g de micélio.

Em cada grupo, os animais receberam, individualmente, os seguintes tratamentos, de abril a junho de 2002: grupo $1-10 \mathrm{~g}$ de péletes por via oral, semanalmente; grupo $2-10 \mathrm{~g}$ de péletes por via oral, quinzenalmente; grupo $3-0,5 \mathrm{~mL}$ de moxidectin por $25 \mathrm{~kg}$ de peso vivo, por via subcutânea, no início do experimento (abril de 2002); e grupo 4 - controle, não tratado.

Mensalmente, dois caprinos traçadores machos anglo-nubianos, livres de nematóides pelo tratamento com oxfendazol (7,5 $\mathrm{mg}$ por $\mathrm{kg}$ de peso vivo), e com idade em torno de oito meses, foram colocados junto ao rebanho permanente, em cada piquete, por 30 dias, sem 
que nenhum animal tivesse recebido qualquer tratamento fúngico. Após 30 dias, os traçadores foram retirados do piquete e permaneceram por mais 14 dias em boxes individuais, alimentados com feno de leucena (13\%), soja (20\%), milho (47\%), talo de capim (17\%), sal mineral (2\%), sal comum (1\%) e água à vontade. Em seguida, os animais foram sacrificados e necropsiados, de acordo com normas internacionais estabelecidas pela World Association for the Advancement of Veterinary Parasitology (WAAVP), descritas por Vercruyse et al. (2002).

A cada sete dias, fezes dos animais dos grupos permanentes foram coletadas diretamente da ampola retal, para avaliação do OPG e coprocultura (Roberts \& O’Sullivan, 1950). A cada 15 dias, cinco caprinos machos permanentes, de cada grupo, foram pesados para o acompanhamento do desenvolvimento ponderal.

Os dados dos exames de fezes e carga parasitária foram transformados para $\log (x+1)$. Os resultados foram submetidos à análise de variância e teste de Tukey, a $5 \%$ de probabilidade.

\section{Resultados e Discussão}

Houve diferença significativa do OPG, entre os grupos de animais tratados com o fungo $M$. thaumasium (grupos 1 e 2) e com vermífugo (grupo 3), em relação ao OPG dos animais do grupo controle (grupo 4). Os animais que receberam tratamento com fungo, uma vez por semana, apresentaram resultados estatisticamente melhores (Tabela 1). O grupo de animais controle apresentou OPG superior, em relação aos demais grupos.

Foi observado que os animais traçadores, introduzidos no piquete do grupo 1 , apresentaram menor carga

Tabela 1. Média mensal da contagem de ovos por grama de fezes, de caprinos $(n=36)$ dos grupos $1,2,3$ e 4 , de abril a junho de 2002, em Sobral, $\mathrm{CE}^{(1)}$.

\begin{tabular}{ccccc}
\hline Grupo $^{(2)}$ & Abril & Maio & Junho & Média \\
\hline 1 & $489 \mathrm{aA}$ & $284 \mathrm{bA}$ & $387 \mathrm{aA}$ & $386,6 \pm 100 \mathrm{~A}$ \\
2 & $503 \mathrm{aA}$ & $418 \mathrm{aB}$ & $847 \mathrm{bB}$ & $589,3 \pm 100 \mathrm{~B}$ \\
3 & $580 \mathrm{aA}$ & $915 \mathrm{bC}$ & $522 \mathrm{aA}$ & $672,3 \pm 100 \mathrm{~B}$ \\
4 & $1.578 \mathrm{aB}$ & $1.863 \mathrm{aD}$ & $1.269 \mathrm{aC}$ & $1.570 \pm 300 \mathrm{C}$ \\
\hline
\end{tabular}

(1)Médias seguidas por letras iguais, minúsculas nas linhas e maiúsculas nas colunas, não diferem entre si pelo teste de Tukey, a $5 \%$ de probabilidade. ${ }^{(2)}$ Grupo 1: tratamento semanal, com $10 \mathrm{~g}$ de péletes do fungo M. thaumasium; grupo 2: tratamento quinzenal, com $10 \mathrm{~g}$ de péletes do fungo $M$. thaumasium; grupo 3: tratamento com vermífugo moxidectin $0,5 \mathrm{~mL}$ por $25 \mathrm{~kg}$ de peso vivo; grupo 4: controle. parasitária, em todos os meses (Tabela 2). As proporções dos nematóides recuperados foram: Haemonchus contortus 90\%, Trichostrongylus colubriformis 3\%, Trichostrongylus axei 3\%, Oesophagostomum columbianum $2 \%$ e Strongyloides papillosus $2 \%$.

Os animais permanentes do grupo controle não apresentaram ganho de peso. Os animais do grupo 1, do grupo 2 e do grupo 3 tiveram ganho de peso superior aos animais do grupo controle (Tabela 3 ).

O maior ganho de peso e a menor carga parasitária, registrados nos animais tratados com o fungo, demonstram a eficiência em se evitar reinfecção, o que pode ter contribuído para a melhor conversão alimentar dos animais. Alves et al. (2003) constataram que bovinos tratados duas vezes por semana, com $20 \mathrm{~g}$ de péletes com cerca de 2-2,5 g de micélios de $M$. thaumasium, obtiveram $23,6 \mathrm{~kg}$ a mais de ganho de peso, em relação aos bovinos que não receberam fungo.

No presente trabalho, o menor OPG dos animais tratados com fungo uma vez por semana, em relação aos demais tratamentos, pode ser atribuído à redução da disponibilidade de larvas infectantes na pastagem, em consequiência da ação nematófaga do fungo. Araújo et al. (2004) testaram, em bezerros em campo, a ação de péletes de $M$. thaumasium e desse fungo associado à ivermectina, durante a estação chuvosa em Minas Gerais; observaram que não houve diferença na redução do OPG entre animais que receberam o fungo duas vezes por semana, durante seis meses, e animais que receberam o fungo duas vezes por semana, com mais duas doses de ivermectina, e concluíram que a administração de $20 \mathrm{~g}$ de péletes, com 2-2,5 g de micélios de $M$. thaumasium, torna os tratamentos com anti-

Tabela 2. Carga parasitária média de nematóides recuperados dos animais traçadores $(n=8)$, de abril a junho de 2002, em Sobral, $\mathrm{CE}^{(1)}$.

\begin{tabular}{cccr} 
Grupo $^{(2)}$ & Abril & Maio & Junho \\
\hline 1 & $158 \mathrm{aA}$ & $361 \mathrm{bA}$ & $86 \mathrm{aA}$ \\
2 & $184 \mathrm{aA}$ & $419 \mathrm{bA}$ & $222 \mathrm{aB}$ \\
3 & $616 \mathrm{aB}$ & $899 \mathrm{bB}$ & $488 \mathrm{aC}$ \\
4 & $857 \mathrm{aB}$ & $1.786 \mathrm{bC}$ & $1.314 \mathrm{bD}$ \\
\hline
\end{tabular}

${ }^{(1)}$ Médias seguidas por letras iguais, minúsculas nas linhas e maiúsculas nas colunas, não diferem entre si pelo teste de Tukey, a 5\% de probabilidade. ${ }^{(2)}$ Grupo 1: tratamento semanal, com $10 \mathrm{~g}$ de péletes do fungo M. thaumasium; grupo 2: tratamento quinzenal, com $10 \mathrm{~g}$ de péletes do fungo $M$. thaumasium; grupo 3: tratamento com vermífugo moxidectin $0,5 \mathrm{~mL}$ por $25 \mathrm{~kg}$ de peso vivo; grupo 4: controle. 
helmíntico desnecessários. Resultado semelhante foi observado por Alves et al. (2003), que obtiveram redução de $88,8 \%$ do OPG, em bezerros que receberam $20 \mathrm{~g}$ de péletes com 2-2,5 g de micélios de $M$. thaumasium, em relação aos animais que não receberam o fungo. Em condições laboratoriais, Castro (2000) registrou taxa acima de $80 \%$, na redução do número de larvas de ciatostomíneos, e Rodrigues et al. (2001) registraram redução de até $99,2 \%$, em animais tratados, comparados aos do grupo controle.

Melo et al. (2003) demonstraram o sucesso do fungo M. thaumasium em passar pelo trato digestivo de caprinos, sem perder a capacidade predatória. O tempo de passagem do fungo, com formação de armadilhas nas fezes, foi de 21 até 24 horas. Em bovinos, Araújo et al. (1999) observaram que o tempo de passagem do fungo $M$. thaumasium, administrado em péletes com 2-2,5 g de micélios, foi de 15 até 110 horas; na passagem com duração 15 horas, houve o maior isolamento do fungo. Em equiinos, esse tempo foi de 8 até 48 horas (Rédua et al., 2002). O maior ou menor tempo de eliminação do fungo está relacionado ao tempo de excreção, nas diferentes espécies animais (Kolb, 1984).

$\mathrm{Na}$ Nigéria, fezes de caprinos sofrem completa desidratação em 24 horas, na estação seca, e em 3 a 4 dias na estação chuvosa (Chiejina \& Fakae, 1989). Tendo-se em vista a rápida dessecação das fezes de pequenos ruminantes, o período de maior disponibilidade de formas infectantes na pastagem é a época chuvosa. Portanto, no Semi-Árido, a utilização de fungos nematófagos, para evitar ou reduzir a transmissão de nematóides gastrintestinais, deve ocorrer na estação chuvosa.

A peletização do micélio não interfere na ação predatória do fungo e pode ser um método importante

Tabela 3. Média mensal de ganho de peso, de caprinos $(\mathrm{n}=20)$ dos grupos 1, 2, 3 e 4, de abril a junho de 2002, em Sobral, $\mathrm{CE}^{(1)}$.

\begin{tabular}{ccccc}
\hline Grupo $^{(2)}$ & \multicolumn{3}{c}{ Peso mensal } & $\begin{array}{c}\text { Ganho de } \\
\text { peso final }\end{array}$ \\
\cline { 2 - 4 } & Abril & Maio & Junho & $9,1 \mathrm{~A}$ \\
2 & $18,2 \mathrm{aA}$ & $26,5 \mathrm{aA}$ & $27,3 \mathrm{aA}$ & $9,1 \mathrm{~A}$ \\
2 & $18,0 \mathrm{aA}$ & $27,4 \mathrm{aA}$ & $27,1 \mathrm{aA}$ & $6,2 \mathrm{~A}$ \\
3 & $18,0 \mathrm{aA}$ & $23,2 \mathrm{aA}$ & $24,2 \mathrm{aA}$ & $0,0 \mathrm{~B}$ \\
\hline
\end{tabular}

${ }^{(1)}$ Médias seguidas por letras iguais, minúsculas nas linhas e maiúsculas nas colunas, não diferem entre si pelo teste de Tukey, a 5\% de probabilidade. ${ }^{(2)}$ Grupo 1: tratamento semanal, com $10 \mathrm{~g}$ de péletes do fungo M. thaumasium; grupo 2: tratamento quinzenal, com $10 \mathrm{~g}$ de péletes do fungo $M$. thaumasium; grupo 3: tratamento com vermífugo moxidectin $0,5 \mathrm{~mL}$ por $25 \mathrm{~kg}$ de peso vivo; grupo 4: controle. no biocontrole de nematóides (Araújo et al., 2000a, 2000b). Vale ressaltar que o fungo peletizado apresenta aplicação prática, pois pode ser mantido em estoque sob refrigeração, o que aumenta seu potencial de utilização em rebanhos.

Chandrawathani et al. (2003), na Malásia, observaram a eficácia do tratamento com o fungo Duddingtonia flagrans, administrado diariamente a ovinos. Terrill et al. (2004) também encontraram redução das larvas, nas fezes de caprinos infectados predominantemente com H. contortus. Estes autores verificaram, ainda, que o oferecimento diário dos fungos (D. flagrans) foi mais eficaz do que o fornecimento a cada dois ou três dias. No entanto, a freqüência dos tratamentos administrados aos animais neste trabalho promoveu a redução da contaminação da pastagem, principalmente no tratamento semanal.

\section{Conclusão}

O fungo Monacrosporium thaumasium peletizado, em doses semanais de 2 a 2,5 g de micélio, é eficiente no controle de nematóides gastrintestinais de caprinos em regiões semi-áridas.

\section{Agradecimentos}

Ao CNPq, por concessão de bolsa.

\section{Referências}

ALVES, P.H.; ARAÚJO, J.V.; GUIMARÃES, M.P.; ASSIS, R.C.L.; SARTI, P.; CAMPOS, A.K. Aplicação de formulação do fungo predador de nematóides Monacrosporium thaumasium (Drechsler, 1937) no controle de nematóides de bovinos. Arquivo Brasileiro de Medicina Veterinária e Zootecnia, v.55, p.568573, 2003.

ARAÚJO, J.V.; FREITAS, B.W.; VIEIRA, T.C.; CAMPOS, A.K. Avaliação do fungo predador de nematóides Duddingtonia flagrans sobre larvas infectantes de Haemonchus contortus e Strongyloides papillosus de caprinos. Revista Brasileira de Parasitologia Veterinária, v.15, p.76-79, 2006.

ARAÚJO, J.V.; GUIMARÃES, M.P.; CAMPOS, A.K.; SÁ, N.C.; SARTI, P.; ASSIS, C.L. Control of bovine gastrointestinal nematode parasites using pellets of the nematode-trapping fungus Monacrosporium thaumasium. Ciência Rural, v.34, p.457-463, 2004.

ARAÚJO, J.V.; SAMPAIO, W.M.; VASCONCELOS, R.S.; CAMPOS, A.K. Effects of different temperatures and mineral salt on pellets of Monacrosporium thaumasium - a nematode-trapping fungus. Veterinarsk Arhiv, v.70, p.181-190, 2000 b. 
ARAÚJO, J.V.; STEPHANO, M.A.; SAMPAIO, W.M. Effects of temperature, mineral salt and passage through the gastrointestinal tract of calves on sodium alginate formulation of Arthrobotrys, a nematode-trapping fungus. Revista Brasileira de Parasitologia Veterinária, v.9, p.55-59, 2000a.

ARAÚJO, J.V.; STEPHANO, M.A.; SAMPAIO, W.M. Passage of nematode-trapping fungi through the gastrointestinal tract of calves. Veterinarsk Arhiv, v.69, p.69-78, 1999.

CASTRO, A.C. Avaliação de fungos Deuteromycetos sobre fases pré-parasíticas de Cyathostominae (Nematosa-Strongylidae). 2000. 100p. Dissertação (Mestrado) - Universidade Federal Rural do Rio de Janeiro, Seropédica.

CHANDRAWATHANI, P.; JAMNAH, O.; WALLER, P.J.; LARSEN, M.; GILLESPIE, A.T.; ZAHARI, W.M. Biological control of nematode parasites of small ruminants in Malaysia using the nematophagous fungus Duddingtonia flagrans. Veterinary Parasitology, v.117, p.173-183, 2003.

CHIEJINA, S.N.; FAKAE, B.B. The ecology of infective larvae of bovine gastrointestinal trichostrongylids in dry season contaminated pastures in the Nigerian derived savanna. Journal for Helminthology, v.63, p.127-139, 1989.

DRECHSLER, C. Some Hyphomycetes that prey on free-living terricolous nematodes. Mycologia, v.29, p.447-552, 1937.

ECHEVARRIA, F.; BORBA, M.F.S.; PINHEIRO, A.C.; WALLER, P.J.; HANSEN, J.W. The prevalence of anthelmintic resistance in nematode parasite of sheep in Southern Latin America: Brazil. Veterinary Parasitology, v.62, p.199-206, 1996.

GITHIGIA, S.M.; WALLER, P.J.; HANSEN, J.W. Impact of gastrointestinal helminths on production in goats in Kenya. Small Ruminants Research, v.42, p.21-29, 2001.

GOMES, A.S.; ARAÚJO, J.V.; RIBEIRO, R.C.F. Differential in vitro pathogenicity of predatory fungi of the genus Monascrosporium for phytonematodes, free-living nematode and parasitic nematodes of cattle. Brazilian Journal of Biology Research, v.32, p.79-83, 1999.

GORDON, H.; WHITLOCK, H.V. A new technique for counting eggs in sheep faeces. Journal of the Council for Science and Industry Research, v.12, p.50-52, 1939.

HAY, F.S.; WALLER, P.J.; HANSEN, J.W.; LARSEN, M. Infestation of sheep dung by nematophagous fungi and implications for the control of free-living stages of gastro-intestinal nematode. Veterinary Parasitology, v.70, p.247-254, 1997.
KOLB, E. Fisiologia veterinária. Rio de Janeiro: Guanabara Koogan, 1984. 486p.

MELO, L.M.; BEVILACQUA, C.; ARAÚJO, J.V.; MELO, A.C.F. Atividade predatória do fungo Monacrosporium thaumasium contra o nematóide Haemonchus contortus, após passagem pelo trato gastrintestinal de caprinos. Ciência Rural, v.33, p.169-171, 2003.

MOTA, M.A.; CAMPOS, A.K.; ARAÚJO, J.V. Controle biológico de helmintos parasitos de animais: estágio atual e perspectivas futuras. Pesquisa Veterinária Brasileira, v.23, p.93-100, 2003.

OVER, H.J.; JANSEN, J.; OLM, P.W. van. Distribution and impact of helminth diseases of livestock in developing countries. New York: FAO, 1992, 221p.

RÉDUA, C.R.O.; SICILIANO, S.; MUJICA, F.; ARAUJO, J.V.; RODRIGUES, M.L.A. Passagem do fungo nematófago Monacrosporium thaumasium pelo trato gastrointestinal de eqüinos. Ciência Animal, v.12, p.127-130, 2002.

ROBERTS, F.H.S.; O’SULLIVAN, J.P. Methods for egg counts and larval cultures for strongyles infesting the gastro-intestinal tract of cattle. Australian Journal of Agriculture Research, v.1, p.99102,1950

RODRIGUES, M.L.A.; CASTRO, A.A.; OLIVEIRA, C.R.C.; ANJOS, D.H.S.; BITTENCOURT, V.R.E.P.; ARAÚJO, J.V. Trapping capability of the Arthrobotrys spp. and Monacrosporium thaumasium on cyathostome larvae. Revista Brasileira de Parasitologia Veterinária, v.12, p.51-54, 2001.

TAYLOR, M.A.; HUNT, K.R.; GOODYEAR, K.L. Anthelmintic resistance detection methods. Veterinary Parasitology, v.103, p.183-194, 2002.

TERRIL, T.H.; LARSEN, M.; SAMPLES, O.; HUSTED, S.; MILLER, J.E.; KAPLAN, R.M.; GELAYE, S. Capability of the nematode-trapping fungus Duddingtonia flagrans to reduce infective larvae of gastrointestinal nematodes in goat feces in the Southeastern United States: dose titration and dose time interval studies. Veterinary Parasitology, v.120, p.285-296, 2004.

VERCRUYSE, J.; HOLDSWORTH, P.; LETONJA, T.; CONDER, G.; AMAMOTO, K.; OKANO, K.; REHBEIN, S. International harmonisation of anthelmintic efficacy guidelines. Veterinary Parasitology, v.103, p.277-297, 2002.

WALLER, P.J.; ECHEVARRIA, F.; EDDI, C.; MACIEL, S.; NARI, A.; HANSEN, J.W. The prevalence of anthelmintic resistance in nematode parasite of sheep in Southern Latin America: general review. Veterinary Parasitology, v.62, p.181-187, 1996.

Recebido em 30 de maio de 2007 e aprovado em 2 de julho de 2007 